\title{
Survey Methods for Assessment of Citrus tristeza virus Incidence in Citrus Nurseries
}

\author{
G. Hughes, Institute of Ecology and Resource Management, University of Edinburgh, West Mains Road, EH9 3JG, \\ Scotland, UK; and T. R. Gottwald, USDA, Agricultural Research Service, Ft. Pierce, FL 34945
}

\begin{abstract}
Hughes, G., and Gottwald, T. R. 2001. Survey methods for assessment of Citrus tristeza virus incidence in citrus nurseries. Plant Dis. 85:910-918.

Monitoring of plant health takes place in citrus nurseries to prevent the distribution of infected plants to commercial groves. In this article, both analytical and simulation methods are used to characterize schemes by which such monitoring may be carried out, in the particular context of Citrus tristeza virus infection. Two aspects of such schemes are discussed in detail. The inclusiveness of a sample is an assessment of the degree of redundancy that occurs because, in some samples, the progeny of identically infected propagation material may appear more than once. The operating characteristic function shows the probability of reaching a decision, based on sampling, that a population of daughter plants has an incidence of infection less than or equal to some adopted threshold level for any actual level of incidence in the population. If the same proportion of the population is assessed at different population sizes, both the inclusiveness and the operating characteristic function vary with population size. However, sample sizes may be calculated so that a specified operating characteristic function is maintained as population size varies. The sample sizes required to meet the conditions specified on the operating characteristics do not increase proportionally with population size. Under such a scheme, fewer samples might need to be taken from large populations of daughter plants than would be the case if a constant percentage sampling scheme were adopted.
\end{abstract}

Additional keywords: budwood, indoor container system, precision

Monitoring the health of plants grown in citrus nurseries for distribution to commercial groves is a basic preventative measure employed for the management of many citrus pathogens. Growers regularly remove dead, declining, or nonproductive trees in mature citrus plantings and replant with young trees. If infected nursery trees are used to replace trees removed from mature plantings, an opportunity is created for the introduction of a pathogen that may not have been present in the original planting, but can now move into the existing trees from the replants (8).

Commercial propagation of citrus usually involves the budding (grafting) of a desirable scion variety onto a rootstock selected for its horticultural and disease resistance properties. Rootstocks are typically produced from seed and budded when

Corresponding author: G. Hughes

E-mail: ghughes@srv0.bio.ed.ac.uk

Accepted for publication 11 April 2001.

Publication no. D-2001-0620-01R

This article is in the public domain and not copyrightable. It may be freely reprinted with customary crediting of the source. The American Phytopathological Society, 2001. a stem diameter of 0.5 to $1.0 \mathrm{~cm}$ is achieved. Budwood is obtained from selected "mother" trees or from budwood increase nurseries. Several thousand buds can be cut from a large mother tree, while individual budwood increase plants may yield 50 to 100 buds per year. In either case, the logistics of producing the millions of nursery trees required in countries with large citrus industries involve use of multiple budwood sources. The entire propagation process from rootstock seeding to marketable "daughter" tree usually takes about 18 to 24 months, but trees may be held in nurseries longer, depending on sales and delivery dates.

Various methods are employed to try to ensure that the nursery trees produced and sold are free of graft-transmissible pathogens (5). While testing of individual trees just prior to sale would be the most accurate approach, it is generally not practical because of the time and cost involved. The common approach is to bud young seedlings (that are assumed to have remained infection-free) using budwood either from mother trees that have undergone virus testing at intervals (in order to provide some assurance that they were not infected at the time budwood was cut) or from designated budwood increase nurseries. Bud- wood increase nurseries are propagated from tested budwood sources and can usually be used for only a limited period of time, during which it is expected that levels of pathogen ingress will be low. Budwood increase nurseries may be tested at intervals to monitor ingress of natural infection or propagation of unrecognized infections in the original budwood source from which the increase nursery material was established.

Mother trees may be isolated from known sources of infection and/or grown in screen houses. However, chances remain for infections that can be substantially increased in subsequent propagation operations. Recent infections in large mother trees may be irregularly distributed and so escape detection. Accordingly, there is a need for sampling protocols that can detect low levels of infection in citrus nurseries. These protocols should address both conventional field nursery production systems, where tree position is fixed from the time of budding until sale, and indoor container production systems (2), where trees are grown in individual containers that may be moved repeatedly during their time in the nursery.

From a sampling perspective, we consider the problem of detecting infection that might arise in a population of daughter plants if budwood had been taken from a large mother tree in which a recent infection had escaped detection because it was not yet systemic. In this scenario, the budwood takes the form of "budsticks," and infection is systemic at the level of the stick, so that all the buds on any one stick are either infected or healthy. In the illustrative examples presented in this article, the population comprises the progeny of $N$ budsticks, each of which provided $n$ buds (all of which subsequently become viable daughter plants), resulting in $n \cdot N$ daughter plants. In the present study, we do not consider sources of variation in $n$.

In a field nursery system, where the rootstock seedlings are budded sequentially along rows, the situation is straightforward because the progeny of a single budstick will naturally be grouped together. If each budstick is assumed to contribute $n=10$ buds, sampling systematically from every tenth daughter plant along a row amounts, 
in effect, to conducting a census of the population at the level of the budstick. Thus, a $10 \%$ sampling rate ensures that the progeny of each budstick feature in the sample. This is the method currently used.

The sampling method currently used for indoor container nursery systems is similar to that used in field nurseries. Material is sampled from every tenth daughter plant on a nursery bench. However, in an indoor container production system, plants are grown in individual containers that may be moved around the benches on which they are kept, both during the budding process and in subsequent agronomic operations. It is not possible to identify a group of daughter plants as the progeny of a particular budstick by their location on a nursery bench. Benches vary in size but typically may accommodate several hundred containers.

For the purpose of monitoring plant health in a nursery using the indoor container system, we assume a simple random sample (SRS) of $m$ daughter plants is drawn from the population comprising the progeny of $N$ budsticks, each of which provided $n$ buds. Material from the sampled plants is then assayed for infection. As an example, suppose that an SRS consists of material taken from $m=5$ daughter plants out of a population of 50 plants, the progeny of $N=5$ budsticks. The total possible number of ways of drawing such a sample is

$$
\left(\begin{array}{c}
50 \\
5
\end{array}\right)=50 !(5 ! \cdot(50-5) !)=2,118,760
$$

Since the population includes the progeny of five sticks, and the sample comprises material from five daughter plants, there is only one kind of sample that has all five budsticks represented-that which has drawn (at random) one daughter plant originating from each budstick. There are, however, 10 daughter plants from each budstick that may be drawn in the sample, so the total number of samples that have all sticks represented is $10^{5}=100,000$. The proportion of samples that have the progeny of all sticks represented is therefore $100,000 / 2,118,760=0.047$. On average, slightly less than $5 \%$ of samples will include progeny of all budsticks when a $10 \%$ sampling rate is applied to populations of 50 daughter plants that are the progeny of five budsticks. It is immediately clear that sampling in indoor container nurseries is a very different proposition from sampling in field nurseries.

Bearing this illustration in mind, we consider characteristics of sampling schemes for citrus nurseries using the indoor container production system. We illustrate more generally the calculation of the extent to which, on average, all the different budsticks used to produce a population of daughter plants are represented in a sample drawn at random from that popu- lation. We outline the calculation of the operating characteristic function, showing (for our purposes here) the probability of reaching a decision, based on sampling, that a population of daughter plants has an incidence of infection less than or equal to some adopted threshold level, for any actual level of incidence in the population.

We are not concerned here with the description of sampling schemes that meet specific regulatory scenarios. Rather, we are concerned with guidelines for the development of such schemes. These guidelines are illustrated using both analytical and simulation methods. The context in which this work was undertaken was the assessment of Citrus tristeza virus (CTV) incidence in citrus nurseries, and the principal objective was to provide an efficient and practical basis for monitoring of the CTV status of citrus nursery trees.

\section{MATERIALS AND METHODS}

Inclusiveness of a sample. Inclusiveness is an issue when budsticks are systemically infected. In the indoor container system, sampling will involve a degree of redundancy - that is, in some samples, daughter plants that are among the progeny of the same budstick will appear more than once. The simple introductory illustration given above does not generalize to situations in which $N$, the number of budsticks, is not the same as $m$, the number of daughter plants sampled, so an alternative method of calculating inclusiveness is required. Using some basic methods in combinatorics (7), it is possible to calculate the number of samples in which, on average, the progeny of at least one budstick are not represented. As an illustration, we re-work the example used above in the introductory section. Consider, then, an SRS of $m=5$ plants taken from a population of $n \cdot N=50$ daughter plants that are known to be the progeny of $N=5$ budsticks, each of which provided $n=10$ buds. The movement of containers around benches that occurs during and after propagation, although not a true randomization, means that it is reasonable to assume that daughter plants from a budstick are not clustered. We refer to the five budsticks that provided the buds as $A, B, C, D$, and $E$, although of course the progeny of these sticks cannot be distinguished from one another at the time of sampling. Let $V$ be the set of all samples with the progeny of at least one budstick not represented. The set $V$ is made up of subsets $V_{A}, V_{B}, V_{C}$, $V_{D}$, and $V_{E}$, which are void of representation of budsticks $A, B, C, D$, and $E$, respectively. The number of elements in set $V$, denoted $\#(V)$, is calculated using the "inclusion-exclusion principle" as follows. Begin by summing the number of elements in each of the subsets:

$$
\#\left(V_{1}\right)=\left[\#\left(V_{A}\right)+\cdots+\#\left(V_{E}\right)\right]
$$

Next, calculate

$$
\#\left(V_{2}\right)=\left[\#\left(V_{A} \cap V_{B}\right)+\cdots+\#\left(V_{D} \cap V_{E}\right)\right]
$$

(i.e., considering all intersections of two subsets),

$\#\left(V_{3}\right)=\left[\#\left(V_{A} \cap V_{B} \cap V_{C}\right)+\cdots+\#\left(V_{C} \cap V_{D} \cap V_{E}\right)\right]$ (i.e., considering all intersections of three subsets), and finally

$$
\#\left(V_{4}\right)=\left[\begin{array}{l}
\#\left(V_{A} \cap V_{B} \cap V_{C} \cap V_{D}\right)+\cdots \\
+\#\left(V_{B} \cap V_{C} \cap V_{D} \cap V_{E}\right)
\end{array}\right]
$$

(i.e., considering all intersections of four subsets). Note that there is no sample that can have all five sticks not represented (i.e., $\#\left(V_{5}\right)=0$ ). For a population of 50 daughter plants derived from five budsticks, each providing 10 buds, the number of samples of five plants with the progeny of at least one stick not represented is then:

$$
\#(V)=\#\left(V_{1}\right)-\#\left(V_{2}\right)+\#\left(V_{3}\right)-\#\left(V_{4}\right)
$$

Further, for a population of 50 daughter plants derived from five budsticks, each providing 10 buds, the number of samples of five plants with the progeny of exactly one stick not represented is:

$$
\#(V[1])=\#\left(V_{1}\right)-\left(\begin{array}{l}
2 \\
1
\end{array}\right) \#\left(V_{2}\right)+\left(\begin{array}{l}
3 \\
1
\end{array}\right) \#\left(V_{3}\right)-\left(\begin{array}{l}
4 \\
1
\end{array}\right) \#\left(V_{4}\right)
$$

Similarly, the number of samples with exactly two sticks not represented is:

$$
\#(V[2])=\#\left(V_{2}\right)-\left(\begin{array}{l}
3 \\
2
\end{array}\right) \#\left(V_{3}\right)+\left(\begin{array}{l}
4 \\
2
\end{array}\right) \#\left(V_{4}\right)
$$

the number of samples with exactly three sticks not represented is:

$\#(V[3])=\#\left(V_{3}\right)-\left(\begin{array}{l}4 \\ 3\end{array}\right) \#\left(V_{4}\right)$

and the number of samples with exactly four sticks not represented is:

$\#(V[4])=\#\left(V_{4}\right)$

More generally, for a population of $n \cdot N$ daughter plants derived from $N$ budsticks, each providing $n$ buds, the number of samples of $m$ plants with the progeny of at least one stick not represented is:

$$
\begin{aligned}
\#(V) & =\left[\#\left(V_{A}\right)+\#\left(V_{B}\right)+\cdots+\#\left(V_{N}\right)\right] \\
& -\left[\#\left(V_{A} \cap V_{B}\right)+\#\left(V_{A} \cap V_{C}\right)+\cdots+\#\left(V_{N-1} \cap V_{N}\right)\right] \\
& +\left[\#\left(V_{A} \cap V_{B} \cap V_{C}\right)+\#\left(V_{A} \cap V_{B} \cap V_{D}\right)+\right] \\
& \vdots \\
& +\cdots+\#\left(V_{N-2} \cap V_{N-1} \cap V_{N}\right) \\
& +\left[(-1)^{N+1} \#\left(V_{A} \cap V_{B} \cdots \cap V_{N}\right)\right]
\end{aligned}
$$

in which the second line of the formula indicates that all intersections of two subsets are considered, the third line indicates that all intersections of three subsets are considered, and so on. The number of 
samples with exactly $k$ sticks $(k=1, \ldots, N)$ not represented is:

$$
\begin{gathered}
\#(V[k])=\#\left(V_{k}\right)-\left(\begin{array}{c}
k+1 \\
k
\end{array}\right) \#\left(V_{k+1}\right)+\left(\begin{array}{c}
k+2 \\
k
\end{array}\right) \#\left(V_{k+2}\right) \\
\cdots+(-1)^{N-k}\left(\begin{array}{l}
N \\
k
\end{array}\right) \#\left(V_{N}\right)
\end{gathered}
$$

Simulations for inclusiveness. In addition to analytical results obtained as described above, sampling simulations were carried out using Minitab for Windows Release 12.1 (Minitab Inc., State College, PA). These simulations were straightforward in design. For example, the case (discussed above) when an SRS of $m=5$ plants is taken from a population of $n \cdot N=$ 50 daughter plants that are known to be the progeny of $N=5$ budsticks, each of which provided $n=10$ buds, is modeled by the Minitab code:

MTB $>$ SET C1

DATA $>(1: 5) 10$

DATA $>$ END

MTB > SAMPLE 5 C1 C2

The first line of code denotes the column of the worksheet that is used to store the "population" from which the sample will be drawn; the second line defines that population as $1,1,1,1,1,1,1,1,1,1,2,2,2,2$, $2,2,2,2,2,2, \ldots, 5,5,5,5,5,5,5,5,5,5$. Symbolically, the line following the SET command has the form $(1: N) n$. The third line indicates the end of data. The fourth line causes an SRS of five (symbolically, $m$ ) individuals to be drawn from the population in the first column and placed in the second column of the worksheet. The sampling is done without replacement.

For each population of interest in the current study, a simulation comprised 100 batches of 100 samples of size $m$. For each batch of 100 samples, the frequency distribution of the number of samples with the progeny of $1,2,3, \ldots, N$ budsticks represented was compiled. Using the results from all 100 batches, the characteristics of these frequency distributions were summarized by calculating the median number of samples (out of 100) with the progeny of $1,2,3, \ldots, N$ budsticks represented. The variability observed in a simulation was characterized by calculating the minimum, 25th percentile, 75th percentile, and maximum number of samples (out of 100) with the progeny of $1,2,3, \ldots, N$ budsticks represented.

Operating characteristic functions. The calculations above, relating to inclusiveness, do not take into account whether or not any of the budsticks were infected. Now consider the problem when there may be $d=0,1, \ldots, N$ infected budsticks among those from which the $n \cdot N$ daughter plants are produced. As previously, we deal with infection that is systemic at the level of the budstick, but not at the level of the mother tree. Each stick provides the same number (n) of buds, and it is assumed that as a result of normal agronomic operations, the progeny of a budstick are not clustered. The number of infected daughter plants is $n \cdot d$. The sample size (number of daughter plants sampled) is $m$. The (hypergeometric) probability distribution of $X$, the number of infected plants in the sample, is given by:

$\operatorname{Pr}(X=x)=h(n \cdot N, m, n \cdot d, x)$

where

$h(n \cdot N, m, n \cdot d, x)=\frac{\left(\begin{array}{c}n \cdot d \\ x\end{array}\right) \cdot\left(\begin{array}{c}n \cdot N-n \cdot d \\ m-x\end{array}\right)}{\left(\begin{array}{c}n \cdot N \\ m\end{array}\right)}$

and $x=0,1, \ldots, m$

The operating characteristic function shows the probability that the observed number of infected plants $(X)$ in a sample will be less than or equal to an adopted threshold value $(c)$ for different actual levels of infection. The probability of obtaining $c$ or fewer infected daughter plants in a sample when the actual incidence of infection (on a proportion scale) is $(n \cdot d) /(n \cdot N), d=0,1, \ldots, N$, is given by:

$\operatorname{Pr}(X \leq c)=H(n \cdot N, m, n \cdot d, c)$

in which

$H(n \cdot N, m, n \cdot d, c)=\sum_{x=0}^{c} \operatorname{Pr}(X=x)$ and $\operatorname{Pr}(X=x)$

is based on equation 5. The operating characteristic function may be used to describe the performance of a specified sampling scheme. For example, for a sampling scheme in which the sample size $(m)$ is defined as a percentage of the population size $(n \cdot N)$, the corresponding operating characteristic is then obtained from equation 6 for given values of population size and threshold $c$.

Alternatively, one can define the performance that one desires from a sampling scheme and calculate the sample size required for this performance to be achieved. In statistical quality control (6), this would usually be done by specifying two points on the desired operating characteristic, such that $\operatorname{Pr}(X \leq c)=1-\alpha$ when the actual incidence (on a proportion scale) is $\left(n \cdot d_{a}\right) /(n \cdot N)\left(d_{a}\right.$ is the corresponding number of infected budsticks), and $\operatorname{Pr}(X \leq c)=\beta$ when the actual incidence (on a proportion scale) is $\left(n \cdot d_{b}\right) /(n \cdot N)\left(d_{b}\right.$ is the corresponding number of infected budsticks). The required minimum sample size $m$ and the corresponding threshold number of infected plants $c$ may then be found from (numerical) solution of the simultaneous equations:

$$
\begin{aligned}
1-\alpha & =H\left(n \cdot N, m, n \cdot d_{a}, c\right) \\
\beta & =H\left(n \cdot N, m, n \cdot d_{b}, c\right)
\end{aligned}
$$

Guenther (3) provides an approximation method for this procedure.
Unfortunately, the methodology leading to equations 7 does not transfer easily to disease management decision making. This is because disease management decision making usually makes reference to only one specific point on the operating characteristic function, at the level of infection (or disease) adopted as the decision threshold. However, an operating characteristic function typically has a value near 0.5 when the actual incidence is near the decision threshold (1) (provided that the threshold is nonzero). We therefore specify a single point on the desired operating characteristic, such that $\operatorname{Pr}(X \leq c)=0.5$ when the actual incidence of infection (on a proportion scale) is at the level defined as the decision threshold, $\left(n \cdot d_{\text {thresh }}\right) /(n \cdot N)$ (where $d_{\text {thresh }}$ is the corresponding number of infected budsticks). The required sample size $m$ may then be found from (numerical) solution of:

$0.5=H\left(n \cdot N, m, n \cdot d_{\mathrm{thresh}}, c\right)$

provided that we define the threshold number of infected plants $c$ in terms of the other parameters. In practice, we can define $c=\left(m \cdot d_{\text {thresh }}\right) /(N)$ (taking the integer part of the expression on the right-hand side). This has the effect of restricting the solutions obtained to those where the incidence of infection in the sample $(\mathrm{c} / \mathrm{m})$ is less than or equal to the incidence of infection (on a proportion scale) adopted as the decision threshold $\left(d_{\text {thresh }} / N\right)$. Among these solutions, it is usually the minimum value of $m$ that is of greatest interest.

A special case occurs when the value of incidence adopted as the decision threshold is zero. In this scenario, we can define the required performance of a sampling scheme by specifying a single point on the desired operating characteristic, such that $\operatorname{Pr}(X \leq c)=\zeta$ is an acceptable probability of a decision, based on sampling, that a population is infection-free, when the actual (nonzero) incidence of infection (on a proportion scale) is $\left(n \cdot d_{z}\right) /(n \cdot N)$ (where $d_{z}$ is the corresponding number of infected budsticks). The required minimum sample size $m$ may then be found from (numerical) solution of:

$\zeta=H\left(n \cdot N, m, n \cdot d_{z}, 0\right)$

In this special case, a good approximate formula is available for minimum $m$ (10):

$m \approx\left(n \cdot N-\frac{n \cdot d_{z}-1}{2}\right)\left(1-\zeta^{\frac{1}{n \cdot d_{z}}}\right)$

Simulations for operating characteristics. As with the study of inclusiveness outlined above, the analytical study of operating characteristic functions was accompanied by simulation studies using Minitab statistical software. The simulations were similar in design to those described above. For example, consider the 
case when there is $d=1$ infected budstick among those from which the 50 daughter plants are produced. As previously, for the purpose of illustration, we deal with infection that is systemic at the level of the budstick (but not at the level of the mother tree) and assume that each stick provides $n=10$ buds. The total number of daughter plants is $n \cdot N(=50)$, the number of infected daughter plants is $n \cdot d(=10)$, and in this illustration, the sample size (number of daughter plants sampled) is $m=5$ (for a $10 \%$ SRS). This is modeled by the Minitab code:

MTB > SET C1

DATA $>(1) 10,(0) 40$

DATA $>$ END

MTB > SAMPLE 5 C1 C2

The first line of code denotes the column of the worksheet that is used to store the "population" from which the sample will be drawn; the second line defines that population of size 50 as $1,1,1,1,1,1,1,1,1,1$, $0,0,0,0,0,0,0,0,0,0, \ldots, 0,0,0,0,0,0,0,0,0,0,0$.

Here, "1" is used to denote "infected", " 0 " is used to denote "uninfected." Symbolically, the line following the SET command

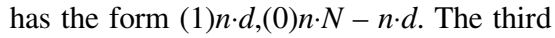
line indicates the end of data. The fourth line causes an SRS of five (symbolically, $m$ ) individuals to be drawn from the population in the first column and placed in the second column of the worksheet. The sampling is done without replacement.

For each simulation, 100 batches of 100 samples of size $m$ were drawn from a population of specified size and incidence of infection. For each batch of 100 samples, the number of samples in which the incidence of infection in the sample was at or below the adopted decision threshold was compiled. Using the results from all 100 batches, the median number of such samples (out of 100) was calculated. The variability observed in a simulation was characterized by calculating the minimum, 25th percentile, 75th percentile, and maximum number of such samples (out of 100).

\section{RESULTS}

Inclusiveness. The number of samples with the progeny of at least one budstick not represented in an SRS of five plants drawn from a population of 50 daughter plants that are the progeny of five budsticks is (from equation 1):

$$
\begin{aligned}
& \#(V)=\left[\left(\begin{array}{c}
40 \\
5
\end{array}\right) \cdot\left(\begin{array}{l}
5 \\
1
\end{array}\right)\right]-\left[\left(\begin{array}{c}
30 \\
5
\end{array}\right) \cdot\left(\begin{array}{l}
5 \\
2
\end{array}\right)\right]+\left[\left(\begin{array}{c}
20 \\
5
\end{array}\right) \cdot\left(\begin{array}{l}
5 \\
3
\end{array}\right)\right] \\
& -\left[\left(\begin{array}{c}
10 \\
5
\end{array}\right) \cdot\left(\begin{array}{l}
5 \\
4
\end{array}\right)\right]=2,018,760
\end{aligned}
$$

As before, the total possible number of such samples is:

$$
\left(\begin{array}{c}
50 \\
5
\end{array}\right)=2,118,760
$$

The proportion of samples with the progeny of at least one stick not represented is therefore 2,018,760/2,118,760 $=0.953$. The complement of this, 0.047 , is the proportion of samples that have the progeny of all sticks represented. That is to say, slightly less than $5 \%$ of samples (on average) will include progeny of all budsticks when a $10 \%$ sampling rate is applied to populations of 50 daughter plants that are the progeny of five budsticks (Fig. 1A).

For an SRS of 10 plants drawn from a population of 50 daughter plants that are the progeny of five budsticks, the number of samples with the progeny of at least one budstick not represented is (from equation $1)$ :

$$
\begin{aligned}
& \#(V)=\left[\left(\begin{array}{l}
40 \\
10
\end{array}\right) \cdot\left(\begin{array}{l}
5 \\
1
\end{array}\right)\right]-\left[\left(\begin{array}{l}
30 \\
10
\end{array}\right) \cdot\left(\begin{array}{l}
5 \\
2
\end{array}\right)\right]+\left[\left(\begin{array}{l}
20 \\
10
\end{array}\right) \cdot\left(\begin{array}{l}
5 \\
3
\end{array}\right)\right] \\
& -\left[\left(\begin{array}{l}
10 \\
10
\end{array}\right) \cdot\left(\begin{array}{l}
5 \\
4
\end{array}\right)\right]=3,939,700,045
\end{aligned}
$$

Now, the total number of possible samples is:

$$
\left(\begin{array}{l}
50 \\
10
\end{array}\right)=10,272,278,170
$$

and so the proportion of samples with the progeny of at least one stick not represented is $3,939,700,045 / 10,272,278,170=$ 0.384 . The complement of this, 0.616 , is the proportion of samples that have the progeny of all sticks represented. That is to say, about $60 \%$ of samples (on average) will include progeny of all budsticks when a $20 \%$ sampling rate is applied to populations of 50 daughter plants that are the progeny of five budsticks (Fig. 1B).

When the population comprises 100 daughter plants that are the progeny of 10 budsticks, the calculations involve numbers that are very large. The total number of possible samples is now

$$
\left(\begin{array}{c}
100 \\
10
\end{array}\right)=1.731 \times 10^{13}
$$

when an SRS of 10 plants is drawn. From equation 3 , the number of such samples with the progeny of at least one stick not represented is $1.730 \times 10^{13}$. The proportion of samples with the progeny of at least one stick not represented is 0.999 . Only $0.1 \%$ of samples will (on average) include progeny of all budsticks when a $10 \%$ sampling rate is applied to populations of 100 daughter plants that are the progeny of 10 budsticks (Fig. 1C). When an SRS of 20 plants is drawn from a population of 100 daughter plants, the total number of possible samples is:

$$
\left(\begin{array}{c}
100 \\
20
\end{array}\right)=5.360 \times 10^{20}
$$

From equation 3 , the number of such samples with the progeny of at least one stick not represented is $3.693 \times 10^{20}$. The proportion of samples with the progeny of at least one stick not represented is 0.689 .
About $30 \%$ of samples will (on average) include progeny of all budsticks when a $20 \%$ sampling rate is applied to populations of 100 daughter plants that are the progeny of 10 budsticks (Fig. 1D).

The distribution of budsticks represented. For an SRS of five plants drawn from a population of 50 daughter plants that are the progeny of five budsticks, $\#(V[1])=900,000, \#(V[2])=967,500$, $\#(V[3])=150,000$, and $\#(V[4])=1,260$ (equation 2). The sum of these is equal to $2,018,760$, as required. The corresponding proportions of sticks not represented in the sample are obtained by dividing \#(V[1]), $\#(V[2]), \#(V[3])$, and $\#(V[4])$ by the total number of samples, in this case:

$$
\left(\begin{array}{c}
50 \\
5
\end{array}\right)=2,118,760
$$

From these proportions, the distribution of number of budsticks represented was calculated (Fig. 1A). (The proportion of samples that have the progeny of all sticks represented is obtained by subtraction, as above.) The modal number of budsticks represented in the sample is three. About $88 \%$ of samples will have three or four sticks represented.

The numbers for an SRS of 10 plants drawn from a population of 50 daughter plants that are the progeny of five budsticks are $\#(V[1])=3,642,945,000, \#(V[2])$ $=294,907,500, \#(V[3])=1,847,540$, and $\#(V[4])=5$. The sum of these is equal to $3,939,700,045$, as required. The corresponding proportions of sticks not represented in the sample are obtained by dividing \#(V[1]), \#(V[2]), \#(V[3]), and \#(V[4]) by the total number of samples, in this case:

$$
\left(\begin{array}{l}
50 \\
10
\end{array}\right)=1.027 \times 10^{10}
$$

From these proportions, the distribution of number of budsticks represented was calculated (Fig. 1B) (the proportion of samples that have the progeny of all sticks represented is obtained by subtraction, as above). The modal number of budsticks represented in the sample is five. About $97 \%$ of samples will have four or five sticks represented.

Using equation 4, equivalent calculations were carried out for SRSs drawn from populations of 100 daughter plants that are the progeny of 10 budsticks. When samples of 10 plants are drawn, the modal number of budsticks represented in the sample is seven, and about $70 \%$ of samples will have six or seven sticks represented (Fig. 1C). When samples of 20 plants are drawn, the modal number of budsticks represented in the sample is nine, and about $77 \%$ of samples will have nine or 10 sticks represented (Fig. 1D).

The results of the simulation studies mimic the analytical results. In all cases, 
the median percentage of samples with $1,2, \ldots, N$ budsticks represented in the sample was close to the analytical result (Fig. 1). The simulation results give some idea of the level of variability likely to be attached to the analytical results in practice.

Operating characteristic functions. To begin, we consider the case where the number of budsticks is $N=5$, of which $d=$ $0,1, \ldots N$ have systemic (at the level of the stick) infections. As previously, each budstick provides $n=10$ buds. The total number of daughter plants is $n \cdot N(=50)$, the number of infected daughter plants is $n \cdot d$ $(=0,10,20,30,40$, or 50$)$. We wish to calculate the probability of reaching a decision, based on sampling, that the population of 50 daughter plants is infection-free, for different actual levels of infection in the population. This is calculated from equation 6 with $c=0$. First, we compare the results for an SRS of $m=5$ plants out of 50 (a 10\% sampling rate) (Fig. 2A) and an SRS of $m=10$ plants out of 50 (a $20 \%$ sampling rate) (Fig. 2B). This simple illustrative example shows, for example, that the probability of concluding that the population of 50 daughter plants contains no infection when one of the budsticks (out of five) was infected is about 0.3 (i.e., about $30 \%$ samples are infection-free) for a $10 \%$ sample (five plants) (Fig. 2A) and about 0.1 (i.e., about $10 \%$ samples are infectionfree) for a $20 \%$ sample (10 plants) (Fig. 2B). The results of the simulation studies again mimic the analytical results. In all cases, the median percentage of infectionfree samples was close to the analytical result (Fig. 2). The simulation results again give some idea of the level of variability likely to be attached to the analytical results in practice.

Next, we consider the effect of different population sizes (i.e., numbers of daughter plants). We calculate the probability of reaching a decision, based on sampling, that a population of $n \cdot N$ daughter plants is infection-free for different actual levels of infection in the population and different numbers of budsticks, $N$ (as before, $n$ is always 10). Again, this is calculated from equation 6 with $c=0$. The different operating characteristics obtained by maintaining a sampling rate of $20 \%$ for populations of $n \cdot N=200,400,600$, and 800 daughter plants are shown in Figure 3. Note that although the percentage sampling rate is constant, the operating characteristic function changes markedly with the population size. For example, with a $20 \%$ sampling rate, the probability of concluding that a population of 200 daughter plants contains no infection when the actual incidence of infection is 0.05 (10 out of 200 budsticks infected) is about 0.1 (i.e., about $10 \%$ samples are infection-free, Fig. 3). Maintaining a $20 \%$ sampling rate, the probabilities of concluding that populations of 400 daughter plants (with 20 infected), 600 daughter plants (with 30 infected), and 800 plants (with 40 infected) contain no infection when the actual incidence of infection is

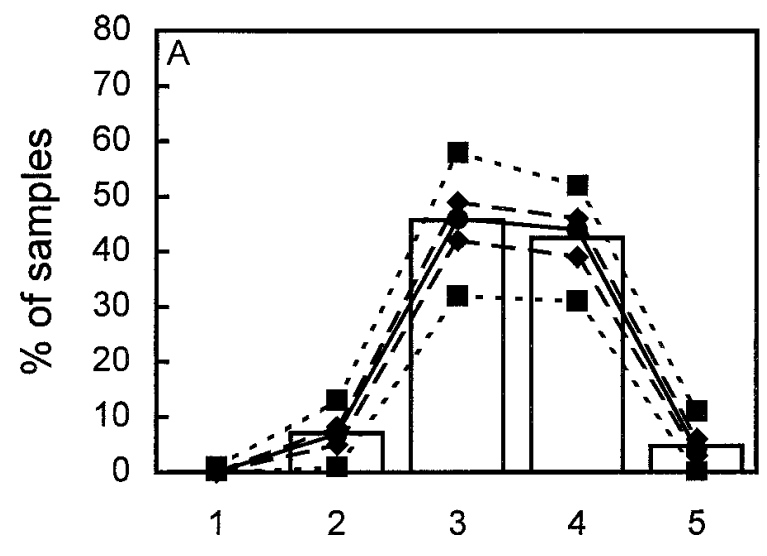

Number of budsticks represented

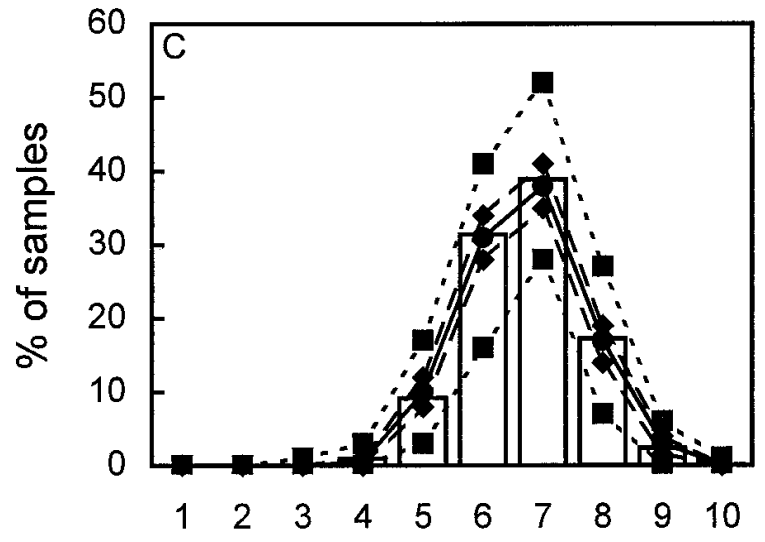

Number of budsticks represented

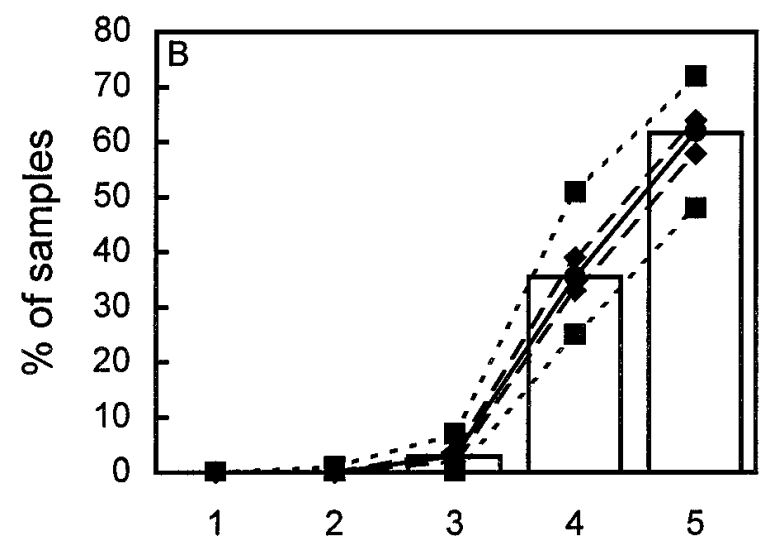

Number of budsticks represented

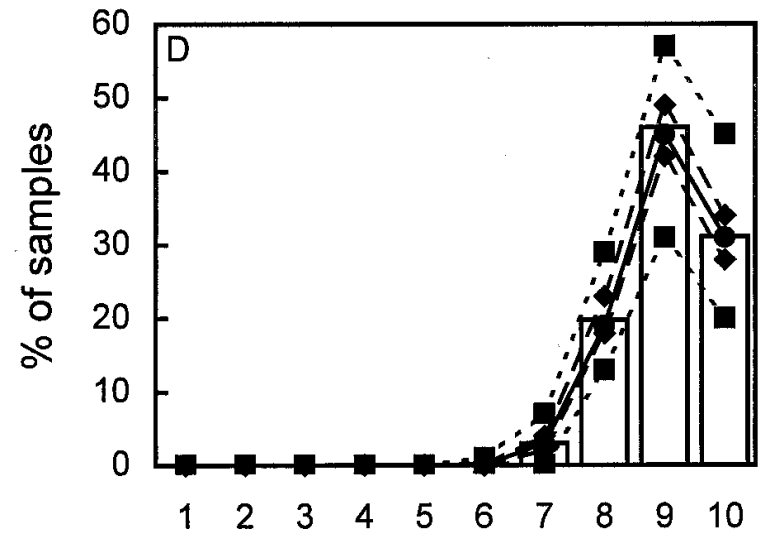

Number of budsticks represented

Fig. 1. Normalized frequency distributions showing the number of budsticks represented in samples drawn from populations of $n \cdot N$ daughter plants that are the progeny of $N$ sticks each providing $n$ buds $(n=10$ in each case). Analytical results are shown as histograms. Simulation results are shown as frequency polygons: $-\longrightarrow$ median of simulation results; - - - -25 th and 75 th percentiles of simulation results; - - - - minimum and maximum of simulation results. Simulation statistics based on 100 batches of 100 samples of the required size. A, $N=5, n \cdot N=50$, sampling rate $=10 \%($ sample size $=$ 5). $\mathbf{B}, N=5, n \cdot N=50$, sampling rate $=20 \%$ (sample size $=10)$. C, $N=10, n \cdot N=100$, sampling rate $=10 \%($ sample size $=10)$. $\mathbf{D}, N=10, n \cdot N=100$, sampling rate $=20 \%($ sample size $=20)$. 
0.05 are, respectively, about $0.01,0.001$, and 0.0001 (Fig. 3).

As an alternative to fixing the sampling rate as a percentage of the population size (and, as shown above, therefore causing the operating characteristic function to vary with population size), we can specify conditions on the operating characteristic and calculate the sample sizes required for the specified conditions to be met at different population sizes. As an initial illustrative example, suppose it was regarded as acceptable that there was probability of 0.1 of reaching a decision, based on sampling, that the daughter plant population was infection-free when in fact 5\% of the population was infected. For any given population size $(n \cdot N)$, the question is then what is the minimum sample size $(m)$ that provides a reasonable solution to equation 9, given the specified conditions. Such solutions must be found by iteration.

In this case, the conditions are that $c=0$ is the adopted threshold number of infected plants, and the probability that a sample is free of infection $\zeta=0.1$ when actual incidence of infection is equal to 0.05 . For a population of $n \cdot N=200$ daughter plants, of which $n \cdot d=10$ are infected, the minimum solution to equation 9 is $m=40$ (this gives $\zeta=0.101$, i.e., about $10 \%$ of samples are infection-free, as specified) (Fig. 4). For populations of 400 daughter plants (with 20 infected), 600 daughter plants (with 30 infected), and 800 daughter plants (with 40 infected), the minimum solutions to equation 9 are, respectively, $m=42(\zeta=0.103)$, $m=43(\zeta=0.101)$, and $m=43(\zeta=0.104)$ (Fig. 4).

Specifying conditions on the operating characteristic and calculating the minimum sample size required to meet these conditions allows essentially the same operating characteristic function to be maintained at different population sizes (Fig. 4). The approximate solutions for minimum sample size obtained from equation 10 were slightly conservative. For populations of 200 daughter plants (with 10 infected), 400 daughter plants (with 20 infected), 600 daughter plants (with 30 infected), and 800 daughter plants (with 40 infected), the solutions to equation 10 were, respectively, $m=41,43,44$, and 44 .

Simulation studies were carried out with populations of 200 individuals (with 10 infected), sample size $m=40 ; 400$ individuals (20 infected), $m=42 ; 600$ individuals (30 infected), $m=43$; and 800 individuals (40 infected), $m=43$. Given the calculations based on equation 9, above, these sample sizes were such that it was expected that, on average, about $10 \%$ of samples would be free of infection. The median percentage of samples free of infection for populations of 200, 400, 600, and 800 individuals, in all of which the actual incidence of infection was 0.05 , were $10,10.5,9$, and $10.5 \%$, respectively. The corresponding intervals between the 25th and 75th percentiles, covering half of the simulations, were 9 to $13 \%, 8$ to $12 \%$, 8 to $12 \%$, and 8 to $12.25 \%$, respectively. Thus, at least in the example investigated,

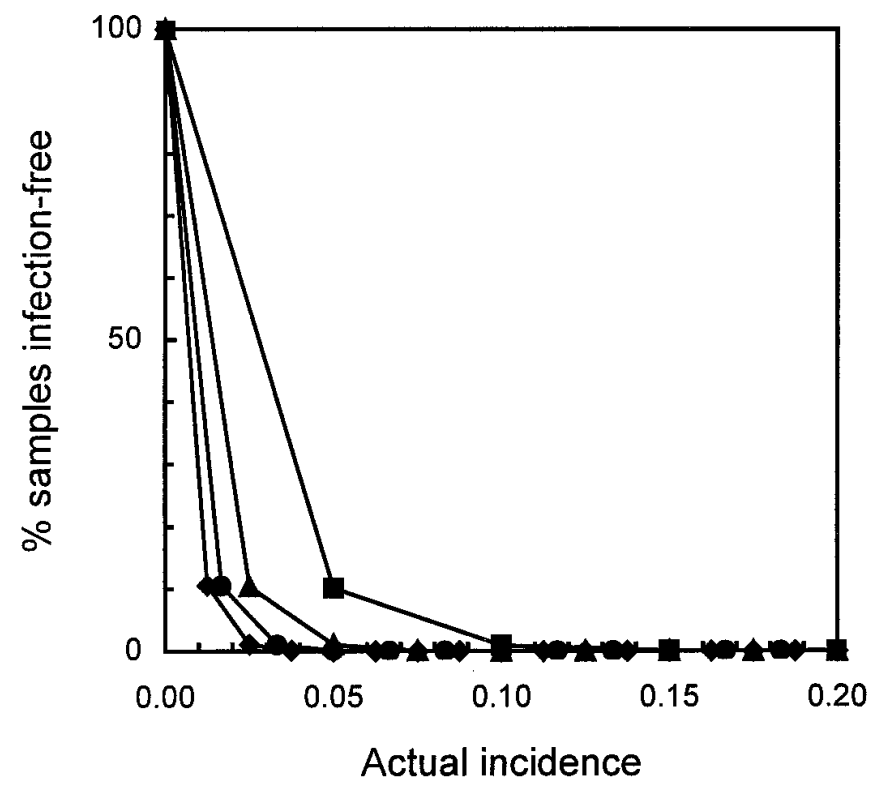

Fig. 3. Operating characteristics showing the percentage of samples free of infection (i.e., no infected daughter plants in sample) for actual levels of incidence of infection in the daughter plant population between zero and 0.2. Analytical results, shown as frequency polygons. In each case, the sampling rate is $20 \%$. The population sizes are: - -200 daughter plants; $-\triangle 400$ daughter plants; $\longrightarrow 600$ daughter plants; $-\longrightarrow 800$ daughter plants.
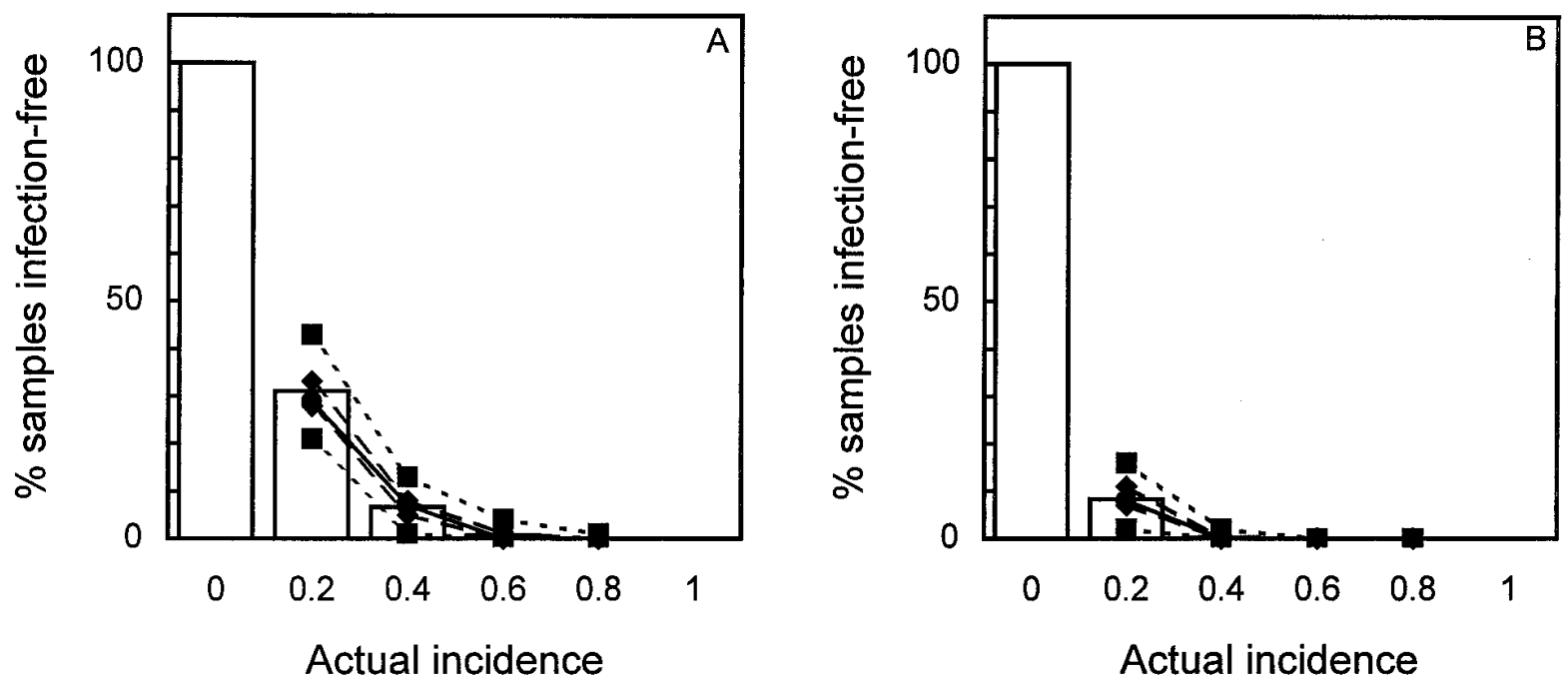

Fig. 2. Operating characteristics showing the percentage of samples free of infection (i.e., no infected daughter plants in sample) for actual levels of incidence of infection in the daughter plant population between zero and one. Analytical results are shown as histograms. Simulation results are shown as frequency polygons: —— median of simulation results; $--\square--25$ th and 75 th percentiles of simulation results; - - $\square$ - - minimum and maximum of simulation results. Simulation statistics based on 100 batches of 100 samples of the required size. A, $N=5, n \cdot N=50$, sampling rate $=10 \%$ (sample size $=$ 5). $\mathbf{B}, N=5, n \cdot N=50$, sampling rate $=20 \%($ sample size $=10)$. 
variability does not appear to increase with population size. Under the specified conditions, the minimum percentage of samples free of infection was $3 \%$, and the maximum percentage free of infection was $18 \%$.

The next illustration also involves specification of conditions on the operating characteristic and calculation of the mini- mum sample sizes required for the specified conditions to be met as population size varies. In this case, we consider an adopted decision threshold that is nonzero. Suppose a decision threshold of 0.05 incidence of infection was adopted. For any given population size $(n \cdot N)$, the question is then what is the minimum sample size $(m)$ that provides a reasonable solution to equation 8 ,

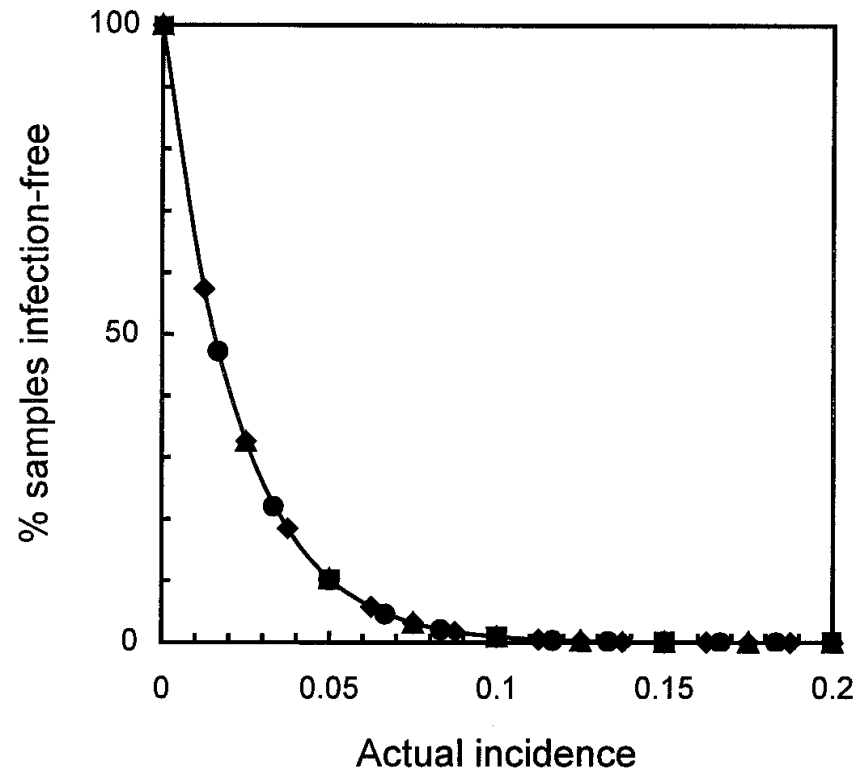

Fig. 4. Operating characteristics showing the percentage of samples free of infection (i.e., no infected daughter plants in sample) for actual levels of incidence of infection in the daughter plant population between zero and 0.2 . Analytical results, based on equation 9 with $\zeta=0.1$ when actual incidence of infection in the daughter plant population is equal to 0.05 . The population sizes are: $-\square-200$ daughter plants (sample size $m=40)$; $-\triangle-400$ daughter plants $(m=42) ;-\bullet 600$ daughter plants $(m=43) ; \longrightarrow-800$ daughter plants $(m=43)$.

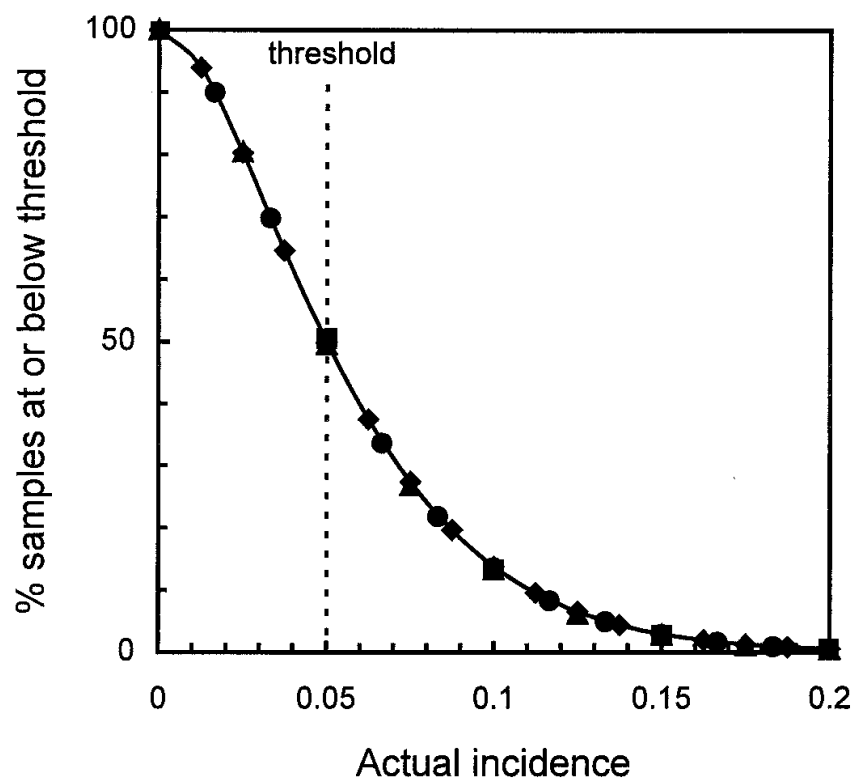

Fig. 5. Operating characteristics showing the percentage of samples at or below the threshold level of incidence $(=0.05)$ for actual levels of incidence of infection in the daughter plant population between zero and 0.2. Analytical results, based on equation 8 with $\operatorname{Pr}(X \leq c)=0.5$ when actual incidence of infection in the daughter plant population is equal to 0.05 . The population sizes are: $-\square-200$ daughter plants (sample size $m=32)$; $-\triangle 400$ daughter plants $(m=33) ;-\bullet 600$ daughter plants $(m=33) ;-\longrightarrow 800$ daughter plants $(m=33)$.

given the specified conditions. Such solutions must be found by iteration.

In this case, the conditions are that $c$ is the integer part of $\left(m \cdot d_{\text {thresh }}\right) /(N)$, where $\left(d_{\text {thresh }} / N\right)$ is the adopted decision threshold on a proportion scale, and that the probability of reaching a decision that a sample contains less than or equal to $c$ infected plants is 0.5 when actual incidence of infection (on a proportion scale) is equal to 0.05 (the adopted decision threshold). For a population of $n \cdot N=200$ daughter plants, of which $n \cdot d=10$ are infected, the minimum solution to equation 8 is $m=32$ (this gives a probability of 0.504 , i.e., about $50 \%$ of samples are at or below the adopted threshold, as specified) (Fig. 5). For populations of 400 daughter plants (with 20 infected), 600 daughter plants (with 30 infected), and 800 daughter plants (with 40 infected), the minimum solutions to equation 8 are, respectively, $m=33$ (probability $=0.495), m=33$ (probability $=0.498$ ), and $m=33$ (probability $=0.500$ ) (Fig. 5). Again, specifying conditions on the operating characteristic and calculating the minimum sample size required to meet these conditions allows essentially the same operating characteristic function to be maintained as the population size varies (Fig. 5).

Simulation studies were carried out with populations of 200 individuals (with 10 infected), sample size $m=32 ; 400$ individuals (20 infected), $m=33 ; 600$ individuals (30 infected), $m=33$; and 800 individuals (40 infected), $m=33$. Given the calculations based on equation 8 , above, these sample sizes were such that it was expected that, on average, about $50 \%$ of samples would be at or below the adopted decision threshold of 0.05 incidence of infection. The median percentages of samples at or below this threshold for populations of 200, 400, 600, and 800 daughter plants, in all of which the actual incidence of infection was 0.05 , were 50 , 50,50 , and $49 \%$, respectively. The corresponding intervals between the 25th and 75th percentiles, covering half of the simulations, were 47 to $53.25 \%, 46.75$ to $53 \%$, 47 to $52.25 \%$, and 47 to $53.25 \%$, respectively. Again, variability does not appear to increase with population size. Under the specified conditions, the minimum percentage of samples at or below the adopted decision threshold of 0.05 was $36 \%$, and the maximum percentage was $62 \%$.

The final set of results concerns solutions to equation 8 that are not the minimum sample sizes. Continuing with the same scenario as outlined for the previous example, we find that for a population of $n \cdot N=200$ daughter plants, of which $n \cdot d=$ 10 are infected, another solution to equation 8 is $m=52$. For populations of 400 daughter plants (with 20 infected), 600 daughter plants (with 30 infected), and 800 daughter plants (with 40 infected), the solutions to equation 8 are, respectively, $m$ 
$=52, m=53$, and $m=53$. Another set of solutions to equation 8 , for the same scenario, is that for populations of 200 daughter plants (with 10 infected), 400 daughter plants (with 20 infected), 600 daughter plants (with 30 infected), and 800 daughter plants (with 40 infected), $m=71,72,72$, and 72 , respectively. The effect of increasing sample size while maintaining the same conditions on the operating characteristic is to increase the (negative) slope of the operating characteristic function at the incidence of infection adopted as the decision threshold (Fig. 6).

\section{DISCUSSION}

The main results illustrated by the examples presented above are as follows. First, not unexpectedly, increasing the percentage sampling rate increases the inclusiveness (compare Figure $1 \mathrm{~A}$ with $1 \mathrm{~B}$ and Figure 1C with 1D) and decreases the probability of a decision, based on sampling, that a population is infection-free when the actual incidence of infection is greater than zero (compare Figure 2A with 2B). Second, if the same percentage sampling rate is kept at different population sizes, the inclusiveness changes (compare Figure $1 \mathrm{~A}$ with $1 \mathrm{C}$ and Figure $1 \mathrm{~B}$ with 1D), as does the operating characteristic function describing (in the example shown) the probability of a decision, based on sampling, that a population is infectionfree when the actual incidence of infection is greater than zero (Fig. 3). Third, sample sizes may be calculated so that a specified operating characteristic function is maintained as population size varies (Figs. 4 and 5). A notable feature of the results is that in the examples presented, the sample sizes required to meet the conditions specified on the operating characteristics do not increase proportionally with population size. This result, which is in fact a general one, means that if an appropriate operating characteristic function can be specified, fewer samples might need to be taken from large populations of daughter plants than would be the case if a constant percentage sampling scheme were adopted.

The key results presented in this article have been produced by simulation methods as well as by mathematical analysis. The simulation studies fulfill a number of purposes. First, the results of the simulation studies were obtained by means of a sampling scheme directly analogous to the physical process envisaged for sampling citrus plants growing in individual containers in an indoor nursery production system. The use of simulation methodology may therefore help to increase the transparency of the results obtained by mathematical analysis as presented above. Second, if any of the assumptions used to develop the mathematical analysis of the indoor container nursery system-for example, that each stick provides the same number of viable buds-were regarded as oversimpli- fications, it may be easier to generate results for more complicated versions of the system by refining the specification of the simulation than by refining the analysis. Thus, presenting the results of the simulation studies here, and showing that they mimic closely the results of the mathematical analysis, has the utility of providing a preliminary validation of the simulation procedures. Third, it is important to realize that operating characteristic functions describe the average performance of sampling schemes. In practice, the operation of a sampling scheme involves variability of performance around that average. Simulation studies help to characterize the likely scale of this variability.

Generally, the operating characteristic function shows the probability of reaching a decision, based on sampling, that a population of daughter plants has an incidence of infection less than or equal to some adopted threshold level, for any actual level of incidence in the population. When the actual level of infection is less than or equal to the adopted threshold value, the operating characteristic shows the probability of a "true negative" (TN) decision, based on sampling. Typically, the probability of a true negative decision is near 1 when the actual incidence is much less than the value adopted as the decision threshold, and declines as actual incidence increases toward the threshold (Fig. 5). When the actual level of infection is greater than adopted threshold value, the operating characteristic shows the probability of a "false negative" (FN) decision, based on sampling. Typically, the probabil- ity of a false negative decision is near 0 (on a proportion scale) when the actual incidence is much larger than the value adopted as the decision threshold, and increases as actual incidence declines toward the threshold (Fig. 5). If the adopted decision threshold is zero, any decision based on sampling that a population is infection-free when the actual level of infection is greater than zero is a false negative (Fig. 4).

A false negative decision is one type of misclassification that may occur when decisions are based on sampling. For nonzero decision thresholds, the other type of misclassification is a "false positive" (FP): a decision based on sampling that the level of infection is greater than the adopted threshold when the actual level of infection is at or below the threshold. The probability of a false positive is obtained as (1 $\operatorname{Pr}[\mathrm{TN}])$. In a quality control context, the precision of a sampling scheme is characterized by the rate of misclassification. The more precise is the sampling scheme, the fewer misclassifications occur. In terms of the operating characteristic, increased precision can be seen as an increase in the (negative) slope of the operating characteristic function at the incidence of infection adopted as the decision threshold (Fig. 6). Thus, the effect of increasing sample size above the minimum required to meet the specified conditions is to increase the precision of sampling. As sample size is increased, the shape of the operating characteristic function more closely resembles that of an ideal operating characteristic function (see Figure 13-3 in literature citation 6).

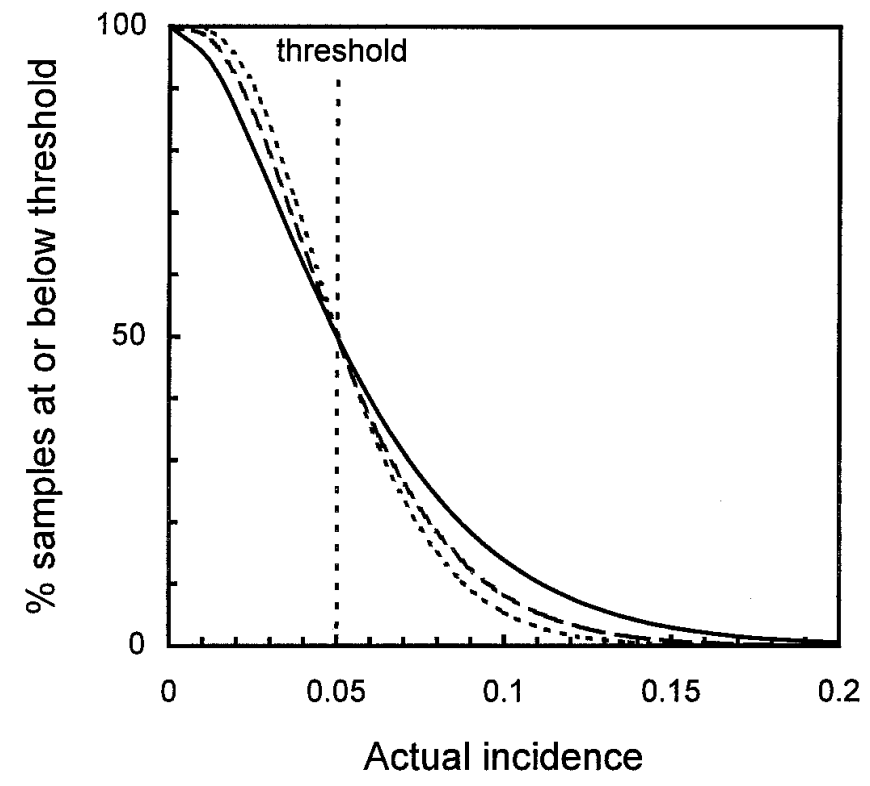

Fig. 6. Operating characteristics showing the percentage of samples at or below the threshold level of incidence $(=0.05)$ for actual levels of incidence of infection in the daughter plant population between zero and 0.2. Analytical results, based on equation 8 with $\operatorname{Pr}(X \leq c)=0.5$ when actual incidence of infection in the daughter plant population is equal to 0.05 . Solid line, population sizes $n \cdot N=200$, 400, 600, 800, (minimum) sample sizes $m=32,33,33,33$, respectively; ——— population sizes $n \cdot N=200,400,600,800$, sample sizes $m=52,52,53,53$, respectively; - - - -, population sizes $n \cdot N$ $=200,400,600,800$, sample sizes $m=71,72,72,72$, respectively. 
In this article, our discussion of the calculation of sample sizes in relation to operating characteristic functions has made the implicit assumption that the subsequent process of detecting the pathogen of concern - in our case, CTV-is free of errors. This may be regarded as reasonable where serological methods are used in laboratory-based assays of plant material collected from nurseries. In cases where this assumption may not be valid-for example, where detection was based on a visual assessment of symptoms associated with infection - a similar analysis could be developed using a "faulty inspection" distribution (4) in place of the hypergeometric. Note also that we have said nothing about whether or not plant material collected by sampling in the nursery and sent to the laboratory for assay will be tested on an individual plant basis or in batches made up of material from groups of plants. In the latter case, methods for group testing (9) may be used to obtain estimates of the incidence of infection at the individual plant scale.

The sampling issues discussed in this article arose in the particular context of CTV-infection in citrus nurseries. Thus, the scenario involves budwood collected from a large mother tree that may have suffered a recent infection that has escaped detection because it is not yet systemic. Such infections are, however, assumed to be systemic at the level of the sticks from which individual buds are taken for grafting onto the rootstock seedlings. The indoor container system for citrus nursery tree production is sufficiently complicated, and variable from operator to operator, that we do not foresee being able to specify a "one size fits all" sampling scheme to meet the requirement for monitoring CTV incidence. However, the mathematical analysis presented does provide general guidelines on how to calculate sample sizes to meet this requirement, provided an operating characteristic function can be specified. The simulation procedures outlined provide a means of describing the characteristics of sampling schemes tailored to meet specific scenarios. Overall, this work also provides a basis for monitoring plant health in citrus nurseries that can be adapted to meet new threats to the industry as they arise in future.

\section{ACKNOWLEDGMENTS}

We thank S. M. Garnsey for many helpful discussions, and the Florida Department of Agriculture and Consumer Services, Division of Plant Industry, and the USDA, Animal and Plant Health Inspection Service, for arranging numerous contacts within the citrus nursery industry and for their encouragement and enthusiasm for this project.

\section{LITERATURE CITED}

1. Binns, M. R., and Nyrop, J. P. 1992. Sampling insect populations for the purpose of IPM decision making. Annu. Rev. Entomol. 37:427453.

2. Castle, W. S., and Ferguson, J. J. 1982. Current status of greenhouse and container production of citrus nursery trees in Florida. Proc. Fla. State Hortic. Soc. 95:42-46.

3. Guenther, W. C. 1973. A sample size formula for the hypergeometric. J. Quality Technol. 5:167-170.

4. Johnson, N. L., Kotz, N., and Sorkin, H. L. 1980. "Faulty inspection" distributions. Commun. Stat. - Theor. Meth. A9:917-922.

5. Koizumi, M. 1998. Production systems of fruit tree nurseries to control grafttransmissible diseases. J. Jpn. Soc. Hortic. Sci. 67:1093-1097.

6. Montgomery, D. C. 1997. Introduction to Statistical Quality Control. 3rd ed. John Wiley $\&$ Sons, New York.

7. Percus, J. K. 1971. Combinatorial Methods. Springer-Verlag, New York.

8. Powell, C. A., and Pelosi, R. R. 1993. Prevalence of severe strains of citrus tristeza virus in Florida citrus nurseries. Hortscience 28:699-700.

9. Rodoni, B. C., Hepworth, G., Richardson, C., and Moran, J. R. 1994. The use of a sequential batch testing procedure and ELISA to determine the incidence of five viruses in Victorian cut-flower Sim carnations. Aust. J. Agric. Res. 45:223-230.

10. Yamamura, K., and Sugimoto, T. 1995. Estimation of the pest prevention ability of import plant quarantine in Japan. Biometrics 51:482490. 